\title{
Combined use of random access and ELISA analyzers in the microbiological serology laboratory
}

\author{
Alessandra Moroni', Antonella Marangoni', Marina Biagi', Silvia Capitani', \\ Enzo Della Bella', Sanzio Ruscello', Franca Savioli', Roberto Cevenini² \\ 'Unità Operativa di Microbiologia, Policlinico S. Orsola-Malpighi, Bologna \\ ${ }^{2}$ Dipartimento Medicina Clinica Specialistica e Sperimentale - Sezione di Microbiologia, Università degli Studi \\ di Bologna, Bologna
}

Key words: Random access analyzers; ELISA tecnique; Serology

Uso combinato di analizzatori ad accesso random ed a micropiastra per la sierologia microbiologica

\section{SUMMARY}

In the last years the trend of centralizing small laboratories in large reference centers led to a careful evaluation of the diagnostic profiles. In the serology laboratory of Microbiology Unit, St. Orsola-Malpighi Hospital, Bologna, Italy the choice has been to combine random access analyzers (ARCHITECT Abbott) and ELISA analyzers (BEPIII Dade Behring).

Received January 16, 2008

Accepted April 15, 2008

\section{INTRODUZIONE}

In questi ultimi anni la tendenza ad accorpare $\mathrm{i}$ piccoli laboratori ha portato ad un aumento del numero di campioni nei grandi centri di riferimento diagnostico e alla necessità di rivedere, ed eventualmente ampliare, la tipologia di offerta dei profili analitici mantenendo alta la qualità del risultato. Nel laboratorio di Sierologia - U.O. Microbiologia-Policlinico S. Orsola-Malpighi di Bologna è stata raggiunta una semplificazione del flusso di lavoro e una riduzione dei tempi di risposta eliminando alcune linee analitiche e potenziando quelle che nel tempo avevano garantito migliore affidabilità diagnostica. L'uso combinato di analizzatori ad accesso random (ARCHITECT Abbott) e a micropiastra (BEPIII Dade-Behring) e di due strumenti dedicati (DELTA Radim per TAS e VIDAS bioMérieux per metodiche ELFA) ha permesso di far fronte al notevole aumento del carico di lavoro e alla riduzione del personale, rendendo più semplici le fasi di addestramento e turnazione.

\section{Presentazione dello Studio}

Nel 1999, con l'acquisizione degli esami per virus epatitici, sifilide e toxoplasmosi, tutta la sierologia microbiologica all'interno del Policlinico S. Orsola-Malpighi è passata di pertinenza dell'Unità Operativa di Microbiologia.

Per fare fronte al progressivo aumento del carico lavoro, mantenere o addirittura ridurre $\mathrm{i}$ tempi di risposta e semplificare la turnazione del personale tecnico, si è tentato nel corso degli anni di accorpare il maggior numero di esami su analizzatori ad accesso random, in particolare AXSYM Abbott, già impiegato per la sierologia dei virus epatitici. Le prove di valutazione delle performance diagnostiche di diversi test e strumenti e della possibilità del loro eventuale utilizzo sono state condotte considerando anche le risorse umane, economiche, e gli spazi disponibili.

Nel 2005 è stato compiuto un passo importante ai fini della riorganizzazione interna spostando l'esecuzione del test di screening per HIV su ARCHITECT Abbott e quello per Sifilide su LIAISON Diasorin (5) rendendo più semplice sia l'esecuzione delle analisi stesse sia la fase preanalitica lavorando direttamente su provetta madre.

Un ulteriore importante snellimento del flusso di lavoro è stato raggiunto nel 2006 quando, con la disponibilità sul mercato anche del test per Sifilide Abbott, tutta la routine per epatite, HIV e Sifilide è passata su ARCHITECT (2), consentendo così l'esecuzione dei tre test dalla stessa provetta madre e la refertazione di esami, spesso richiesti come screening pre-operatorio, in giornata.

Anche per quanto riguarda il gruppo TORCH e le cosiddette sierologie "minori" tra il 2005 e il 2007

\section{Corresponding author: Alessandra Moroni}

Unità Operativa di Microbiologia, Policlinico S.Orsola-Malpighi, - Via Massarenti 9, 40I38 Bologna.

Tel:05। 63645I6; Fax:05। 307397 -E-mail:moroni@med.unibo.it 
si è cercato di automatizzare al massimo l'esecuzione dei test, uniformando le linee analitiche e giungendo alla riduzione del numero di aliquote $\mathrm{e}$ di quantità di siero necessarie.

Dal 2005 anche le indagini per Toxoplasmosi sono state eseguite su analizzatori in micropiastra BEPIII Dade-Behring accorpando così tutte le analisi del gruppo TORCH sulla medesima strumentazione, che negli anni di costante impiego nel nostro laboratorio ha dimostrato maggiore robustezza, affidabilità diagnostica $(1,3-4)$ e capacità di sopportare elevati carichi di lavoro giornaliero.

Nel 2007 questa linea analitica è stata potenziata ulteriormente iniziando a eseguire su BEPIII tutte le analisi per antigeni respiratori, per le quali non esiste al momento possibilità di esecuzione su strumenti ad accesso random.

Sempre nel 2007 con l'impiego del TECAN FE 500 è stata raggiunta l'automazione totale della fase preanalitica (check-in, aliquota e rintracciabilità delle provette madri).

Attualmente il numero di provette che giungono all'Unità Operativa di Microbiologia per esami sierologici ("tappo rosa" per HIV, Epatite, Sifilide e "tappo bianco" per le rimanenti analisi sierologiche) è di circa 400 al giorno. Le provette arrivano da reparti interni e da punti prelievo e strutture esterne dalle 8.00 alle 14.30. La fase preanalitica costituisce un turno settimanale di un operatore tecnico.

Passata la fase di "check-in" la provetta madre "rosa" viene caricata in continuo da 1 operatore tecnico sulla linea analitica Abbott, costituita da tre ARCHITECTi2000 ${ }^{\circledR}$ per un carico totale di 250 provette/giorno e 11 diversi parametri: Sifilide; $\mathrm{HIV} \mathrm{Ab/Ag;} \mathrm{Epatite} \mathrm{B} \mathrm{(HBsAg,} \mathrm{HBsAb,} \mathrm{HBcAb,}$ $\mathrm{HBcAb}$ IgM, HBeAb e $\mathrm{HBeAg}$ ); Epatite $\mathrm{C}$ (HCVAb); Epatite A (HAVAb IgG e HAVAb $\operatorname{IgM})$.

I primi risultati sono disponibili intorno alle $\mathrm{h}$. 10.30 in continuo fino alle 15.30.

Sulla stessa strumentazione vengono eseguiti giornalmente i test sierologici in urgenza per la validazione delle UET (HBsAg; HIV Ab/Ag; $\mathrm{HCV}$ Ab; Sifilide) e in emergenza per i donatori d'organo.

I campioni con risultato ARCHITECT positivo o dubbio vengono testati il giorno successivo con metodiche di conferma, mantenendo tempi di refertazione ridotti.

Dalla provetta "bianca" vengono invece prodotte 3 aliquote: TORCH, "Respiratori", TAS e metodi manuali.

Due operatori tecnici gestiscono la linea analitica Dade-Behring, composta da due diluitori Tecan Genesis RSP200 e RSP100, rispettivamente per
TORCH e "Respiratori”, e quattro BEPIII ${ }^{\circledR}$, per un totale di 105 provette/giorno e 39 diversi parametri: CMV IgG e IgM; EBV IgG e IgM, Rosolia IgG e IgM e Avidity; Toxoplasmosi IgG, IgM e IgA; Helicobacter pylorii IgG; HSV IgG; VZV IgG;

Adenovirus IgG e IgM; Mycoplasma pneumoniae IgG e IgM; Pertosse IgG e IgM; RSV IgG e IgA; Influenza A IgG e IgA; Influenza B IgG e IgA; Parainfluenza IgG e IgA; Chlamydia IgG e IgA; Enterovirus IgG e IgM; Legionella IgG e IgM; Morbillo IgG e IgM; Parotite IgG e IgM; Borrelia burgdorferi IgG e IgM (6).

I risultati della linea analitica Dade-Behring sono disponibili in blocco intorno alle 14.30.

Test di conferma per Toxo IgG, IgM, Toxo Avidity e determinazioni anticorpali in urgenza per CMV e Toxo in donatori d'organo sono eseguite su VIDAS bioMérieux.

La determinazione del Titolo AntiStreptolisinico (TAS) viene effettuata sul nefelometro DELTA Radim (30 campioni/giorno) dallo stesso operatore tecnico che processa in media 10 provette/giorno con metodiche manuali Western Blot (Sifilide IgG e IgM, Borrelia IgG e IgM, Toxo IgG e IgM, H. pylorii), Immunofluorescenza (HHV-6, R. conorii, C. burnetii, Leptospira), Emoagglutinazione Passiva (Ameba, Leishmania, Schistosoma, Tripanosoma, Echinococco), Agglutinazioni (Paul-Bunnel1, TPHA, RPR, VDRL, Criptococco) per un totale di 21 parametri diversi.

\section{CONCLUSIONI}

L'aver automatizzato la fase pre-analitica e la quasi totalità della routine sierologica impiegando due sole linee analitiche principali ha portato $\mathrm{i}$ seguenti vantaggi:

- collegamento in rete con semplificazione della fase di refertazione e annullamento della possibilità di errore dovuta alla refertazione manuale;

- snellimento delle fasi di addestramento del personale tecnico;

- rapidità e uniformità di refertazione;

- riduzione del numero di analisi manuali

e ha permesso di far fronte al notevole aumento del carico di lavoro e alla contemporanea riduzione sia del personale tecnico che degli spazi disponibili.

\section{BIBLIOGRAFIA}

1. Hamkar R, Jalilvand S, Mokhtari-Azad $\mathrm{T}$, et al. Assessment of IgM enzyme immunoassay and IgG avidity assay for distinguishing between primary and secondary immune response to rubella vaccine. $J$ Virol Methods 2005; 130: 59-65.

2. Jonas G, Pelzer C, Beckert C, Hausmann M, Kapprell H. Performance characteristics of the ARCHITECT 
Anti-HCV assay. J Clin Vir 2005; 34: 97-103.

3. Kremer JR, Schneider F, Muller CP. Waning antibodies in measles and rubella vaccinees-a longitudinal study. Vaccine 2006; 24: 2594-601.

4. Lefevre-Pettazzoni M, Bissery A, Wallon M, Cozon G, Peyron F, Rabilloud M. Impact of spiramycin treatment and gestational age on maturation of Toxoplasma gondii immunoglobulin $\mathrm{G}$ avidity in pregnant women. Clin Vaccine Immunol 2007; 14: 239-43
5. Marangoni A Sambri V, Accardo S, et al. Evaluation of LIAISON ${ }^{\circledR}$ Treponema Screen, a novel recombinant antigen-based chemiluminescence immunoassay for the laboratory diagnosis of syphilis. Clin Diagn Lab Immunol 2005; 12: 1231-4.

6. Marangoni A, Moroni A, Accardo S, Cevenini R. Borrelia burgdorferi VlsE antigen for the serological diagnosis of Lyme borreliosis. Eur J Clin Microbiol Infect Dis 2008; In press. 